\title{
Erratum to: A best practice position statement on pregnancy in chronic kidney disease: the Italian Study Group on Kidney and Pregnancy
}

\author{
Gianfranca Cabiddu $^{1} \cdot$ Santina Castellino $^{2} \cdot$ Giuseppe Gernone $^{3} \cdot$ Domenico Santoro $^{4} \cdot$ Gabriella Moroni $^{5}$. \\ Michele Giannattasio $^{6}$ - Gina Gregorini $^{7} \cdot$ Franca Giacchino $^{8} \cdot$ Rossella Attini $^{9} \cdot$ Valentina $^{2}$ Loi $^{1} \cdot$ Monica Limardo $^{10}$. \\ Linda Gammaro ${ }^{11}$ - Tullia Todros ${ }^{9}$ Giorgina Barbara Piccoli ${ }^{12,13}$
}

Published online: 15 June 2017

(C) Italian Society of Nephrology 2017

\section{Erratum to: J Nephrol (2016) 29:277-303 DOI 10.1007/s40620-016-0285-6}

The article A best practice position statement on pregnancy in chronic kidney disease: the Italian Study Group on Kidney and Pregnancy, written by Gianfranca Cabiddu, Santina Castellino, Giuseppe Gernone, Domenico Santoro, Gabriella Moroni, Michele Giannattasio, Gina Gregorini, Franca Giacchino, Rossella Attini, Valentina Loi, Monica Limardo, Linda Gammaro, Tullia Todros, Giorgina Barbara Piccoli was originally published Online First without open access. After publication in volume 29, issue 3, page 277-303 the author decided to opt for Open Choice and to make the article an open access publication. Therefore, the copyright of the article has been changed to $($ The Author(s) [2017] and the article is forthwith distributed under the terms of the Creative Commons Attribution 4.0 International License (http://creativecommons.org/licenses/ by/4.0/), which permits use, duplication, adaptation, distribution and reproduction in any medium or format, as long as you give appropriate credit to the original author(s) and the source, provide a link to the Creative Commons license, and indicate if changes were made.
The online version of the original article can be found under doi:10.1007/s40620-016-0285-6.

Giorgina Barbara Piccoli

gbpiccoli@yahoo.it

Nephrology, Azienda Ospedaliera Brotzu, Cagliari, Italy

2 Nephrology and Dialysis, Taormina Hospital, Taormina, Italy

3 Nephrology, S. Maria Degli Angeli Hospital, Putignano, Italy

4 Nephrology and Dialysis, AOU “G. Martino”, Messina, Italy

5 Nephrology, Fondazione Ca' Granda Ospedale Maggiore, Milan, Italy

6 Dipartimento Nefrodialitico Città di Bari Hospital, Bari, Italy
Nephrology, Spedali Civili di Brescia, Brescia, Italy

8 Nephrology, Ospedale d'Ivrea, Ivrea, Italy

9 Obstetrics, Department of Surgery, University of Torino, Torino, Italy

10 Nephrology, Azienda Ospedaliera della Provincia di Lecco, Lecco, Italy

11 Nephrology, Ospedale Fracastoro, San Bonifacio, Italy

12 Nephrology, ASOU San Luigi, Department of Clinical and Biological Sciences, University of Torino, Turin, Italy

13 Nephrologie, Centre Hospitalier du Mans, Le Mans, France 